\title{
Ultrastructure of the Developing Erythrocytes
}

\author{
Akira B. Miura, Akira Shibata, Tetsuo Akihama, Yasuyuki \\ Endo and Yasuko SaIto \\ First Department of Internal Medicine, Akita University School \\ of Medicine, Akita
}

\begin{abstract}
Miura, A. B., Shibata, A., Agihama, T., Endo, Y. and Saito, Y. Ultrastructure of the Developing Erythrocytes. Tohoku J. exp. Med., 1974, 112 (4), 299-313 - Ultrastructure of the erythroblasts in the bone marrow of human adults was studied. Although the knowledge of the stem cells has been increasing, the precise morphology of them is not yet clearly identified in the bone marrow specimen. However, the so-called proerythroblasts and the developing erythroblasts are easily identified under electron microscopic observations by the characteristic features such as the round shape of the cells, almost perfectly round nucleus with unique chromatin figures, polysome formation of a few ribosomes, a number of microtubules and micropinocytic vesicles, and siderosomes. - ultrastructure of erythroblast; human bone marrow
\end{abstract}

Although some ultrastructural characteristics of the erythroblasts such as micropinocytosis and siderosomes have been noticed, systematic observation on the human bone marrow is rare. On the contrary, a great amount of biochemical informations have been elucidated. The morphological interpretation coincident with the biochemical knowledge of hemoglobin synthesis is difficult at present, but the understanding of erythroblasts will be deepened by collaboration with morphological and biochemical findings.

This report describes the ultrastructural development of the erythroblasts.

\section{Materials and Methods}

The specimens of the bone marrow were obtained from normal individuals by puncture. They were fixed in 2.5 per cent glutaraldehyde with subsequent fixation in 2 per cent osmium tetoxide, dehydrated in graded series of ethanol and embed,ded in Epon mixture. The ultrathin sections were stained with uranyl acetate and lead citrate, and were examined with a JEM 100 B type electron microscope.

\section{RESUlts}

Shape and size of the erythroblasts

The erythroblasts in the early and intermediate stages had a well regulated round or spheroidal shape. The interdigitation or the prominent projection of the cell surface was not observed. The late erythroblasts and the reticulocytes often had a prominent lumpy projection in one side of the cells (Fig. 5). The shape of

Received for publication, October 12, 1973. 
mature erythrocytes was entirely irregular under the electron microscope but the surface membrane was smooth and did not show any complicated interdigitation.

The size of erythroblast decreased with maturation from about $20 \mu \mathrm{m}$ to 10 $\mu \mathrm{m}$ in diameter. Though the area of the mature erythrocytes became small, their shape was so irregular that the diameter could not be measured.

\section{Nucleus}

Almost all of the nucleus of erythroblasts were round or oval. The nucleus showed a smooth margin without prominent projection. It diminished in volume with the maturation.

Nucleolus

A single prominent nucleolus was observed in the early stage in contact with or near the nuclear membrane (Fig. 1). The rest of nucleolus was recognized in the intermediate erythroblasts.

\section{Nucleoplasm}

In the earliest stage, the nucleoplasm was chiefly composed of euchromatin except for a prominent nucleolus (Fig. 1). The dense chromatin (heterochromatin) increased rapidly as the cell developed. The area of euchromatin decreased with maturation, but the nuclear pores always communicated with the euchromatin even in the latest stage. Intranuclear inclusion bodies were scarcely observed.

\section{Nuclear membrane}

The outer and inner nuclear membranes were sometimes dissociated, and the autophagic vacuolous structure was sometimes seen in this space.

A number of nuclear pores, up to ten or so, were recognized on a profile of each nucleus.

\section{Cytoplasm}

Cytoplasm of the early erythroblast was filled with free ribosomes. The polysome formation of three to six ribosomes became clear in accordance with the cell development. A definite polysome pattern as seen in plasma cells was not clear.

The filamentous or granular substances in the cytoplasm, which were regarded as hemoglobin or their precursor, increased their density according to the development and became nubeculous. The free ribosomes decreased in number in proportion to the increase of hemoglobin concentration, but a number of polysomes remained in the reticulocytes.

The mature erythrocytes showed the homogeneous cytoplasm of high density and had no organelles. 


\section{Mitochondria}

About ten mitochondria, larger ones partly ruptured at the time of fixation and smaller round ones with well preserved inner structure, were observed. The former decreased in number with maturation, and were scarcely seen in the late erythroblasts.

The mitochondria were scattered in the early stage, but a few of the small round ones often gathered to one side of the nucleus in the late erythroblasts (Figs. 3 and 4). Some mitochondria were very closely connected with nucleus.

A small number of iron particles were often observed in the mitochondria, but the occurrence of a large amount of iron particles as was seen in the sideroachrestic anemia was exceptional.

In reticulocytes, mitochondria tended to gather and to increase in electron density to demonstrate degenerative features.

\section{Endoplasmic reticulum}

The endoplasmic reticulum poorly developed in the erythroblasts. In the earliest stage, a small amount of vesicular or short tubular granular endoplasmic reticulum and vesicular or irregular shaped agranular reticulum were recognized, and they decreased in number as the cells developed and were scarcely seen in the late erythroblasts.

The rest of the Golgi complex or newly formed vacuoles at the time of the nuclear extrusion seemed as if they were the endoplasmic reticulum in the late erythroblasts and the reticulocytes.

\section{Centrosomes}

The Golgi complex, mainly composed of vacuoles and vesicles, moderately developed in the early erythroblasts (Figs. 1 and 2). Sometimes the Golgi granules were also recognized, but the membranous structures were rare.

As the cells developed, the Golgi vesicles and granules disappeared. Remains of the Golgi vacuoles were often seen even in the reticulocytes.

The centrioles, paired and close to each other, were seen in the neighborhood of the Golgi complex in the early and intermediate stages (Fig. 2).

Microtubules were often seen in groups near the centrioles (Fig. 2). A few of mitochondria were gathered in the Golgi area.

Dense granules and siderosomes

About ten or so dense granules with a limiting membrane and homogeneous contents, which were regarded as the primary lysosomes and similar to the Golgi granules, were recognized in the early erythroblasts (Figs. 1 and 2). Almost all of these granules diminished in the intermediate stage.

Autophagic vacuoles with irregular or lamellar structure were sometimes seen in the late stage.

The siderosomes with numerous iron particles usually had a limiting membrane (Fig. 8). The iron particles were variant in number and usually distributed equally 
in the siderosome. Sometimes a clear zone was recognized between the dense interior and the limiting membrane.

Siderosomes were seen in one out of several erythroblasts in an ultrathin section. Erythroblasts with two or more siderosomes were sometimes observed in the late stage.

Aggregations of numerous iron particles without a limiting membrane were uncommon (Fig. 9). The electron density of the circumference of such siderosomes was often increased.

Lamellar or myeloid structures in the siderosomes were not unusual in the late stage.

The broken siderosomes discharging the iron particles were never observed.

There was no specific relation between the siderosomes and other organelles. Usually the siderosomes were not clos 3 to mitochondria or coated vesicles containing iron particles, but occasionally the microtubules were seen nearby the siderosomes.

\section{Microtubules}

There were many microtubules in the erythroblasts. They ran straightly or windingly, and in groups or alone. Sometimes they were mixed irregularly (Fig. 10).

A few of short microtubules ran along the nuclear membrane. In the centrosome, ten or so microtubules irregularly ran windingly (Fig. 2). Some of them were recognized near the mitochondria in groups in the late erythroblasts or the reticulocytes. A solitary microtubule without any relation to organelles also ran sporadically.

In the early erythroblasts, the microtubules in the Golgi area were most prominent. After the intermediate stage, variable kinds of microtubules were seen abundantly. In the reticulocytes, they were recognized only near the mitochondria.

Mitotic spindles, which were composed of scores of microtubules forming bundles, often protruded from the cell membrane (Fig. 11). Sometimes they united two erythroblasts in the resting phase (Fig. 12).

\section{Cell surface and micropinocytosis}

Cell surface of the erythroblasts was smooth, and prominent indentation was rare except in the latest stage just before denucleation (Fig. 5).

Attachment structures, such as desmosomes or tight junctions, were never observed.

A number of micropinocytoses were observed on the cell surface in every stage, especially in the late erythroblasts. More than ten micropinocytoses were observed in each cell on a thin section.

A small part of cell surface became thick and curved to inside, and the outside of the thick surface became dark. Sometimes a few iron particles were attached to the thickened area (Fig. 6). 
Micropinocytic vesicles, solitary or in groups up to ten or so in number (Fig. 7), separated from the cell membrane into the cytoplasm, became round, swelled as they went into the inside, and finally ruptured. Release of the iron particles into the cytoplasm at the time of rupture was sometimes seen.

Usually they had no relation to other organelles, but they were often close to mitochondria.

Micropinocytosis, often close to each other, was seen in any area of the cell surface.

The electron density of the contents was almost equal to that of the surroundings, but sometimes clear except just under the vesicle membrane.

There was no evidence that the erythroblast directly took the iron particles from the broken reticulum cell.

\section{Drscussion}

The ultrastructure of erythroblasts is characterized by the spherical nucleus showing a unique chromatin distribution, polysomes decreasing with and hemoglobin increasing with development of the cell, a variety of microtubules, micropinocytosis, coated vesicles and siderosomes, making easy to distinguish them from other cells.

Although the stem cell of blood cells cannot be identified at present, the erythroblast is easily distinguished even in an early stage from the myeloblast by its remarkable characteristics.

Although it was assumed that the reticulum cell situated in the center of the erythroblasts island might be the stem cell, the reticulum cell is in fact a well-differentiated cell, and cannot be considered to become the erythroblast because there is no morphological transition between the two. The erythroblasts island is deemed to play a role in the process of denucleation or supplying certain substances to the erythroblasts. On the basis of findings that in the erythroblast island the reticulum cell collapsed and released iron particles and that adjacent erythroblasts directly took them in, the process was called "rhopheocytosis" (Policard and Bessis 1958). Some authors observed that, also in yolk sac hematopoiesis (Sorenson 1961) and hepatic hematopoiesis (Sorenson 1960), iron particles were given directly to the erythroblasts from adjacent cells.

However, Tanaka et al. (1966) noted that such rhopheocytosis was not seen even in a hypersideremic state. Also in our observations, mutual exchange of iron between reticulum cell and erythroblast could not be found at all, and we assumed that iron particles may usually be taken from the plasm by the erythroblasts.

Concerning micropinocytosis and coated vesicles a detailed description by Fawcett is available (Fawcett 1965). The coated vesicles are assumed to be involved in a mechanism of intake of large molecular substances and are said to be found in various cells, especially in the erythroblasts among marrow cells.

Intake of iron particles by micropinocytosis is often observed, but frequently there is also micropinocytosis involving no iron particles, suggesting that other large molecular substances are taken into the cell in a similar manner. Tanaka 
et al. (1966) insisted that the ferritin is synthesized on the erythroblast membrane. The coated vesicles, as they move away from the cell membrane, gradually swell and rupture to release the substances taken in. On the other hand, there is an opinion. that these findings may be related reversely to release of substances out of the cell (Zail et al. 1964), but it will be reasonable to consider that intake of substances happens.

A small number of iron particles can also be found in the mitochondria and the cytoplasmic matrix. Siderosomes, aggregations of a large number of iron particles, can be seen in every stage from early erythroblast to reticulocyte and most frequently in the intermediate stage. Normally, siderosomes are found in only two to three out of ten erythroblasts on ultrathin sections, and the erythroblast with two or more siderosomes is rare. Regarding the form of siderosomes, a detailed description is available (Bessis and Breton-Gorius 1962). They are classified into four types. They are mostly enveloped by a limiting membrane with a large number of iron particles contained therein, whereas groups of iron particles without any boundary or siderosomes with some structure other than iron particles are rather exceptional. As to the growth and fate of the siderosomes and their participation in hemoglobin synthesis, there are many unknown points. Though there is a report that in early erythroblasts free ferritin molecules are dispersed throughout the cytoplasm (Bessis 1967), we have never obtained such a finding.

The most characteristic finding of the ultrastructure of erythroblast is the development of microtubules. In the 19th century, tubular elements were found in the nucleated cell of chick embryo and were called "Randreifen" (Dehler 1895). They were studied in detail by means of supravital staining and were assumed to be related to maintenance of a biconvex shape of erythroblast (Meves 1911). In 1959, Fawcett observed microtubules under an electron microscope in toad-fish erythrocytes and assumed them to be what had been described as Randreifen (Fawcett 1959). Later, the same view was obtained by observing erythrocytes of amphibia and reptilia (Maser and Philpott 1964). However, insofar as our observations on the human erythroblast are concerned, the microtubules are often isolated, and it is questionable whether they correspond with Randreifen demonstrated by supravital staining.

The function of microtubules is not known well. Maser and Philpott (1964) presumed that microtubules were related to motion and asymmetry and in particular to maintenance of the biconvex shape of erythrocytes. However, such presumption is also questionable because erythroblasts are lower in motility than other blood cells and no more microtubules can be found in mature erythrocytes showing the most irregular form, and they are abundantly recognized in nearly globular intermediate erythroblasts. In view of the variety of their form and their close correlation with other organelles as observed frequently, the function of the microtobules may not be uniform.

The mitotic spindle shows a structure similar to the microtobules, being readily distinguishable by its unique shape. The remnant of mitotic spindle can be seen 
frequently even in the intermediate erythroblasts, and a mitotic spindle is found, though rarely, to be binding two late erythroblasts. A similar structure was found also in the erythroblasts of a human fetus liver and regarded as cilia (Fukuda and Sato 1971), but it is deemed to be the remnant of mitotic spindle.

Mitochondria of the early erythroblast are irregular in shape and often large in size, usually showing a trend of rupturing in the process of the fixation. This finding is also recognized in the fixation only with osmium tetroxide. Such large mitochondria appear rarely in the late erythroblasts, and small, round mitochondria with well preserved internal structure are noticeable instead.

Mitochondria taking part in heme-synthesis are considered to belong to the small round type. This is supported by the finding that in the case of sideroblastic anemia with a failure in heme-synthesis, a great number of iron particles are found in this type of mitochondria.

Any attachment structure between adjacent cells cannot be observed. A desmosome-like structure was noted between erythroblast and tissue cell in the primitive blood forming tissue of avian yolk sac (Edmonds 1966) and human fetus (Fukuda and Sato 1971), but its occurrence may be related to the field or the stage of genesis of erythroblasts.

Denucleation of the erythroblasts can hardly be observed in the normal human marrow. According to a microcinema view, erythroblasts fluctuate intensively in trying to release nuclei, but the duration of this movement is quite short (Bessis and Bricka 1952). According to observations on anemic dogs (Simpson and Kling 1967), plethoric mice (Orlic et al. 1965) and synergic cells (Skutelsky and Danon 1967), nuclei are pushed out through a narrow passage, and a few of vesicles and mitochondria gather near the nuclear membrane on the cytoplasm-rich side, and vesicles spread out to separate nuclei. Conspicuous ruffling of the cell membrane and unilateral gathering of organelles seen in the late erythroblasts and the reticulocytes can be considered to represent processes directly before and after denucleation.

\section{References}

1) Bessis, M. (1967) Morphology of the different stages of maturation of the cells of the erythrocytic series in mammals. Exp. Biol. Med., 1, 220-233.

2) Bessis, M.C. \& Breton-Gorius, J. (1962) Iron metabolism in the bone marrow as seen by electron microscopy: A critical review. Blood, 19, 635-663.

3) Bessis, M. \& Bricka, M. (1952) Aspect dynamique des cellules du sang. Son étude par la microcinématographie en contraste de phase. Rev. Hemat. 7, 407-435.

4) Dehler, A. (1895) Beitrag zur Kenntnis des feineren Banes der roten Blutkörperchen beim Hühnerembryo. Arch. mikr. Anat., 46, 414 430.

5) Edmonds, R.H. (1966) Electron microscopy of erythropoiesis in the avian yolk sac. Anat. Rec., 154, 785-806.

6) Fawcett, D.W. (1959) Electron microscopic observations on the marginal band of nucleated erythrocytes. Anat. Rec., 133, 379.

7) Fawcett, D.W. (1965) Surface specialization of absorbing cells. J. Histochem. Cytochem., 13, 75-91.

8) Fukuda, T. \& Sato, H. (1971) Desmosomes, cilia and peculiar structure of membranes in erythroblasts of human fetal liver. Virchow. Arch. Abt. B., 7, 309-313.

9) Maser, M.D. \& Philpott, C.W. (1964) Marginal bands in nucleated erythrocytes. 
Anat. Rec., 150, 365-382.

10) Meves, F. (1911) Gesammelte Studien an den roten Blutkörperchen der Amphibien. Arch. mikr. Anat., 77, 465-540.

11) Orlic, D., Gordon, A.S. \& Rhodin, J.A.G. (1965) An ultrastructural study of erythropoietin-induced red cell formation in mouse spleen. J. Ultrastruct. Res., 13, 516-542.

12) Policard, A. \& Bessis, M. (1958) Sur un mode d'incorporation des macromolècules par la cellule, visible au microscope èlectronique: la rhopheocytose. Acad. Sci., 246, 3194-3197.

13) Simpson, C.F. \& Kling, J.M. (1967) The mechanism of denucleation in circulating erythroblasts. J. Cell Biol., 35, 237-245.

14) Skutelsky, E. \& Danon, D. (1967) An electron microscopic study of nuclear elimination from the late erythroblast. J. Cell Biol., 33, 625-635.

15) Sorenson, G.D. (1960) An electron microscopic study of hematopoiesis in the liver of the fetal rabbit. Amer. J. Anat, 106, 27-40.

16) Sorenson, G.D. (1961) An electron microscopic study of hematopoiesis in the yolk sac. Lab. Invest., 10, 178-193.

17) Tanaka, Y., Brecher, G. \& Bull, B. (1966) Ferritin localization on the erythroblast cell membrane and ropheocytosis in hypersiderotic human bone marrows. Blood, 28, 758-769.

18) Zail, S.S., Charlton, R.W., Torrance, J.D. \& Bothwell, T.H. (1964) Studies on the formation of ferritin in red cell precursors. J. clin. Invest., 43, 670-680.

Figs. 1 and 2. Early erythroblast. Euchromatin and nucleolus are prominent. Golgi complex is moderately developed. Some winding microtubules run near the Golgi area. Lysosomes are recognized sporadically. $\times 12,000, \times 30,000$. 


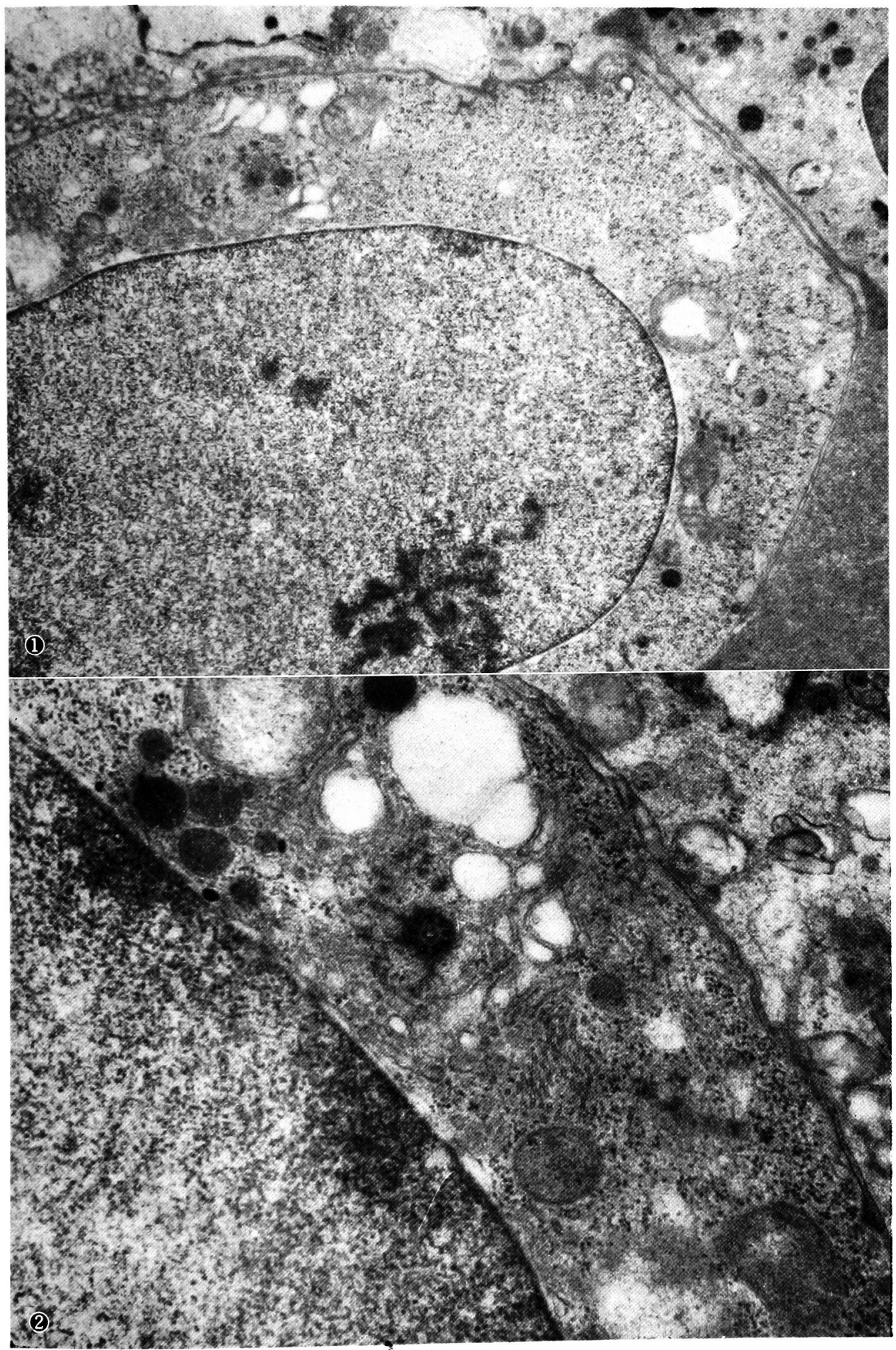


Fig. 3. Intermediate erythroblast with decreasing number of ribosomes and increasing concentration of hemoglobin. $\times 9,500$.

Fig. 4. Reticulocyte. Mitochondria and vesicles gather at one side. $\times 10,000$.

Fig. 5. Late erythroblast. Probably directly before denucleation. Cell surface at one side prominently ruffled. $\times 18,000$. 


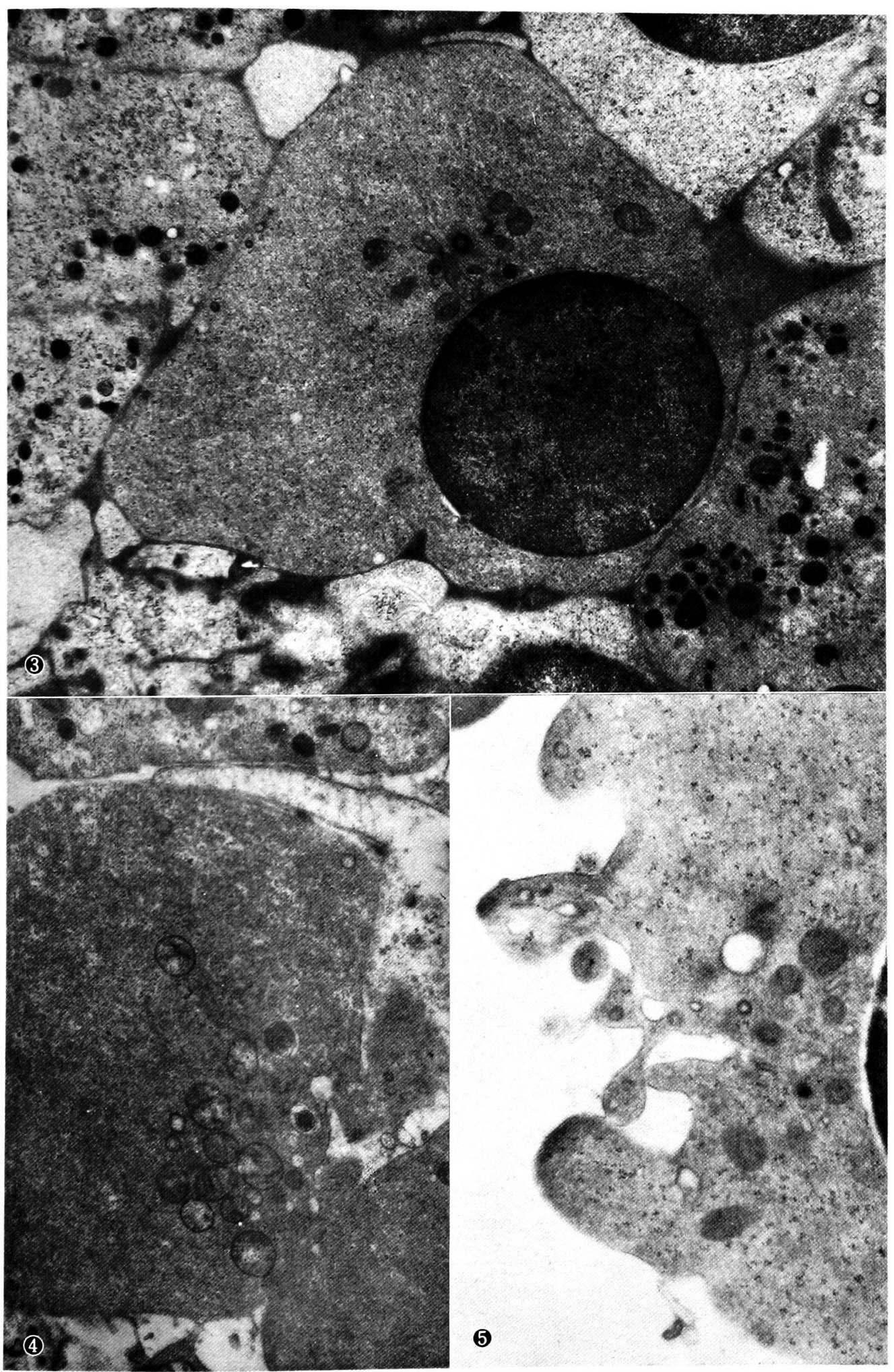


Fig. 6. A few of micropinocytic vesicles with or without iron particles. $\times 28,000$.

Fig. 7. Aggregation of micropinocytic vesicles. Some become swollen and ruptured. $\times 55,000$.

Figs. 8 and 9. Siderosomes with or without limiting membrane. $\times 18,000, \times 26,000$. 


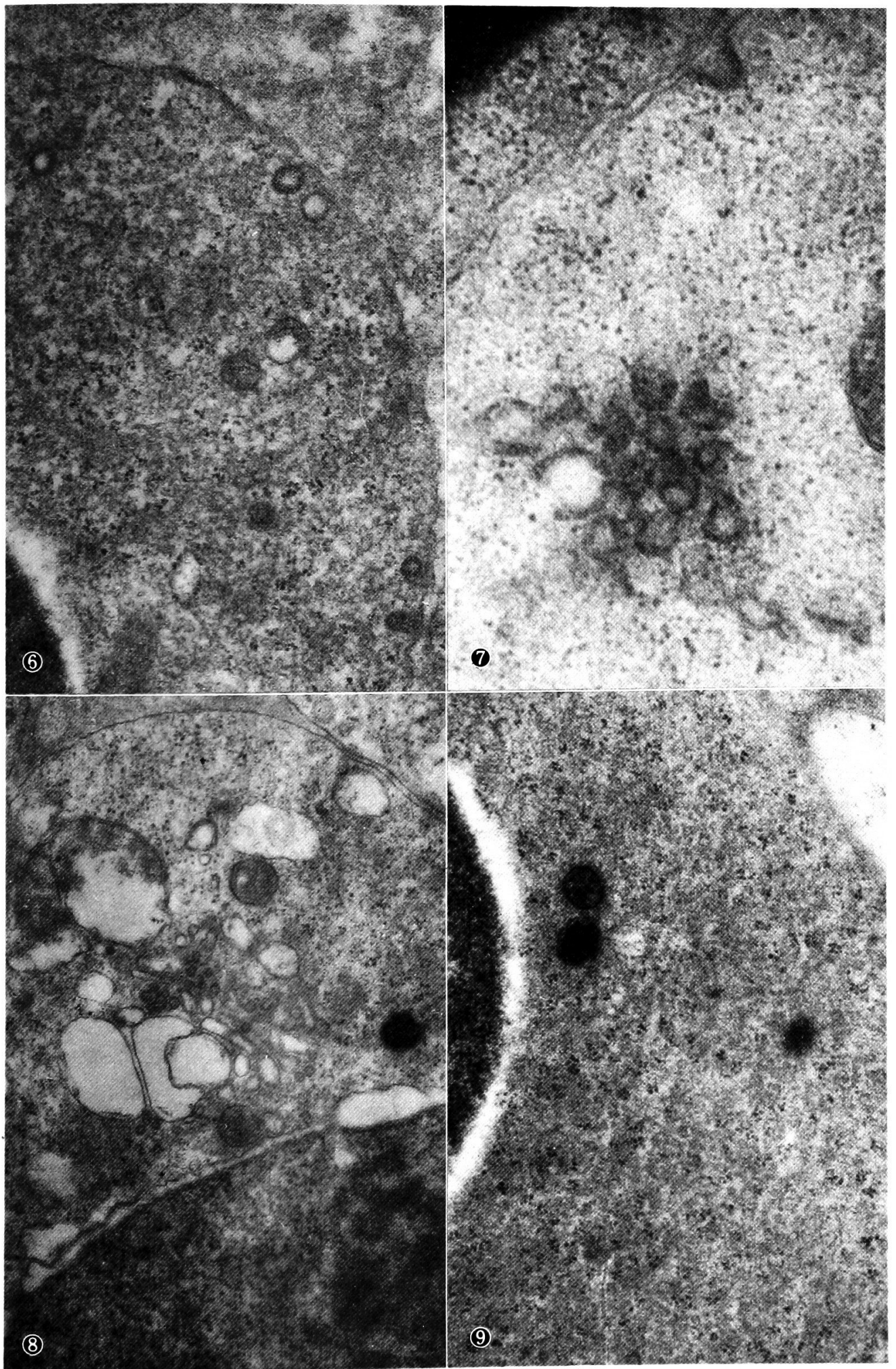


Fig. 10. Many microtubules, long or short, run separately. $\times 40,000$.

Fig. 11. Rest of mitotic spindle protrudes beyond cell surface. $\times 18,000$.

Fig. 12. Rest of mitotic spindle connects two intermediate erythroblasts. $\times 30,000$. 


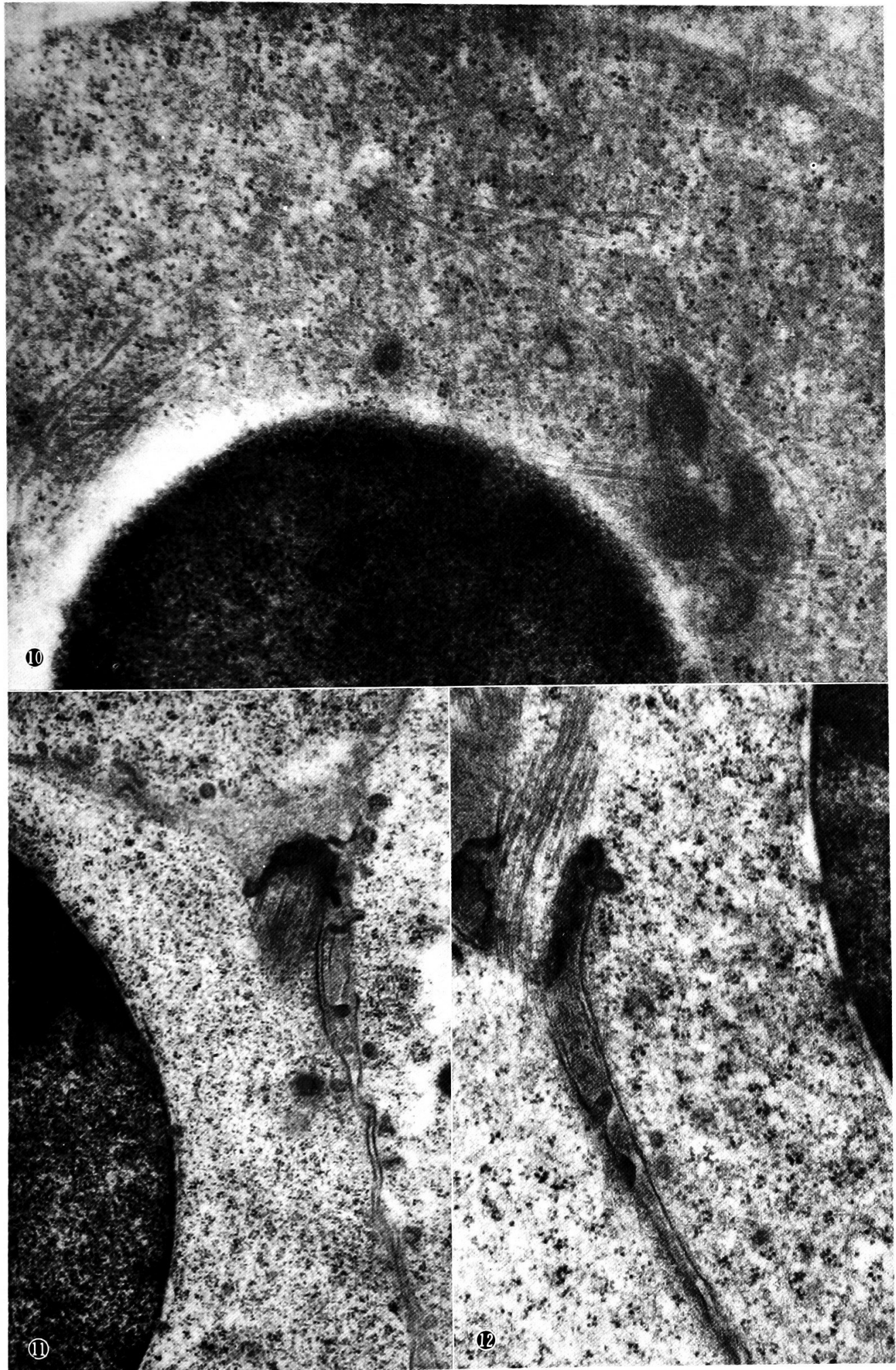

